\title{
Burnout in Young Family Physicians: Variation Across States
}

\author{
Anna Hansen, BA, Lars E. Peterson, MD, PhD, Bo Fang, PhD, \\ and Robert L. Phillips Jr., MD, MSPH
}

Family physicians 3 years out of training report high rates of emotional exhaustion and depersonalization, symptoms of burnout, with considerable variation between states. High rates of burnout among new family physicians is concerning and significant state-level variation suggests that state-related factors may contribute to or reduce burnout. (J Am Board Fam Med 2018;31:7-8.)

Keywords: Family Physicians, Professional Burnout

Burnout refers to the psychological exhaustion resulting from long-term stress, and it puts physicians at risk for poor mental health, decreased productivity, and abandonment of career medicine. ${ }^{1}$ The progression of burnout has been defined in stages, beginning with emotional exhaustion, followed by depersonalization toward others, and eventually resulting in loss of professional achievement and subsequent desire to leave medicine. ${ }^{2}$ Reported prevalence of burnout in family physicians ranges from $25 \%$ to $63 \%$ with the highest prevalence in female physicians under 40 years of age. ${ }^{2-4}$ Developing responses to burnout requires better understanding of context and causes. In this case, our objective was to determine whether state-level variation exists for 2 domains of burnout-emotional exhaustion and

This article was externally peer reviewed.

Submitted 5 July 2017; revised 27 October 2017; accepted 27 October 2017.

From the College of Medicine, University of Kentucky, Lexington, KY (AH); Department of Sociology, University of Kentucky, Lexington (AH); American Board of Family Medicine, Lexington (LEP, BF, RLP).

Funding: AH was supported by the American Board of Family Medicine Foundation.

Conflict of interest: LEP, BF, and RLP are employees of the American Board of Family Medicine.

Corresponding author: Lars E. Peterson, MD, PhD, American Board of Family Medicine, 1648 McGrathiana Parkway, Suite 550, Lexington, KY 40511-1247 (E-mail: lpeterson@theabfm.org).

\footnotetext{
See Related Commentary on Page 5.
}

depersonalization-of recent family medicine graduates. Identifying state-level variation could support further investigation into state-specific policies or other issues that may affect physician burnout.

We used data from a cohort of family medicine residency graduates from 2013 who responded to the inaugural National Graduate Survey in 2016, a survey of American Board of Family Medicine Diplomates who completed residency in 2013. ${ }^{5}$ This survey included 2 validated questions measuring the emotional exhaustion ("I feel burned out from my work") and depersonalization ("I have become more callous toward people since I took this job") domains of burnout. ${ }^{6}$ Each question had the following response options: never, a few times a year or less, once a month or less, a few times a month, once a week, a few times a week, every day. The presence of emotional exhaustion or depersonalization was indicated for responses of once a week or more frequently. We produced state-level averages based on reported state of practice. Due to small sample sizes in some states, we only included states with over 30 respondents. This study was approved by the American Academy of Family Physicians Institutional Review Board.

The overall response rate was $67.8 \%$ (2069 of 3051), and after restricting to states with adequate numbers of respondents our final sample was 1551 physicians in 23 states. The response rate in the included states ranged from $55.7 \%$ to $78.6 \%$. The mean percentage of respondents who reported symptoms of burnout through emotional exhaus- 
Figure 1. Emotional exhaustion and depersonalization rates of 2013 family medicine residency graduates by state of practice in $2016(n=1551)$.

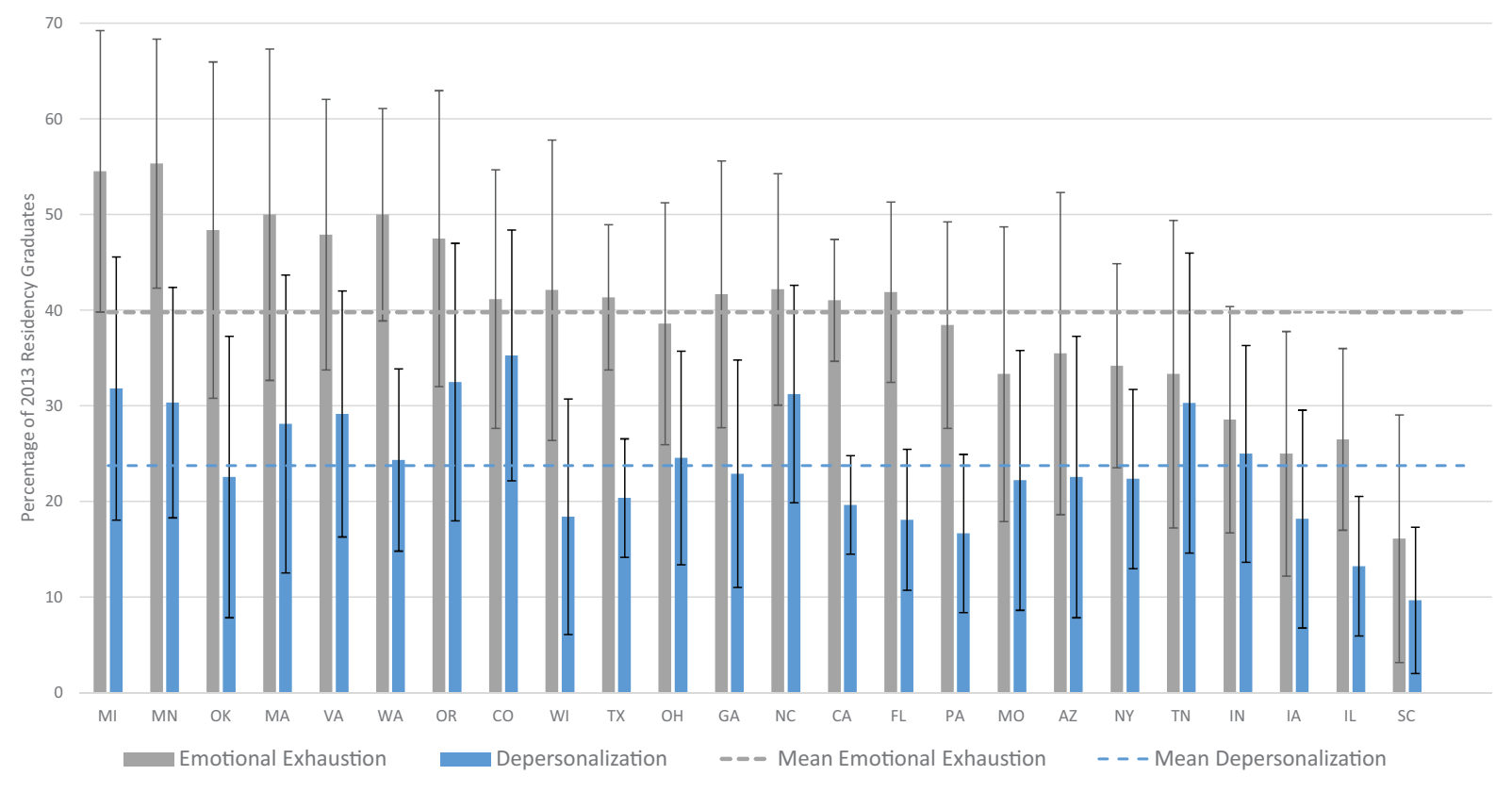

tion and depersonalization were $39.8 \%$ and $23.7 \%$, respectively (see Figure 1). There was considerable variation among states, such that the range around these means was $39.6 \%$ with respect to emotional exhaustion rates, and $20.7 \%$ with respect to depersonalization. Rates of emotional exhaustion and depersonalization did not consistently correspond. In Minnesota, respondents reported the highest rates of emotional exhaustion $(55.4 \%)$, but reported a much lower rate of depersonalization (30.4\%). Respondents in Colorado reported the highest rate of depersonalization (35.3\%), but an emotional exhaustion rate only slightly above the mean (41.2\%). South Carolina reported the lowest rates of both emotional exhaustion (16.1\%) and depersonalization $(9.7 \%)$.

As the US health care system evolves, providers are forced to rapidly adapt to change, resulting in consequences to physician wellbeing, effectiveness, and attitude. ${ }^{1}$ The proportion of young family physicians reporting symptoms of burnout-emotional exhaustion or depersonalization-varies significantly between states, and the relationship between prevalence of emotional exhaustion and depersonalization also varies. Variation among states suggests that there may be state-level factors, such as policies, payer-mix, or even culture that affect burnout. There may be general contributors to physician burnout, but these results indicate that to adequately frame solutions, further exploration of state-level factors is needed.

To see this article online, please go to: http://jabfm.org/content/ 31/1/7.full.

\section{References}

1. Shanafelt TD, Dyrbye LN, West CP. Addressing physician burnout: The way forward. JAMA 2017; 317:901-2.

2. Leiter MP, Maslach C. "Areas of worklife: A structured approach to organizational predictors of job burnout." Emotional and physiological processes and positive intervention strategies. Emerald Group Publishing Limited; 2003, 91-134.

3. Puffer JC, Knight HC, ONeill TR, et al. Prevalence of burnout in board certified family physicians. J Am Board Fam Med 2017;30:125-6.

4. Shanafelt TD, Hasan O, Dyrbye LN, et al. Changes in burnout and satisfaction with work-life balance in physicians and the general US working population between 2011 and 2014. Mayo Clin Proc 2015;90: $1600-13$.

5. Mitchell KB, Maxwell L, Miller T. The National Graduate Survey for Family Medicine. Ann Fam Med 2015;13:595-6.

6. West CP, Dyrbye LN, Sloan JA, Shanafelt TD. Single item measures of emotional exhaustion and depersonalization are useful for assessing burnout in medical professionals. J Gen Intern Med 2009;24: $1318-21$. 